\section{FUNGAL SYMBIOSIS}

\section{Seeing the unseen}

Curr. Biol. 29, 2043-2050 (2019).

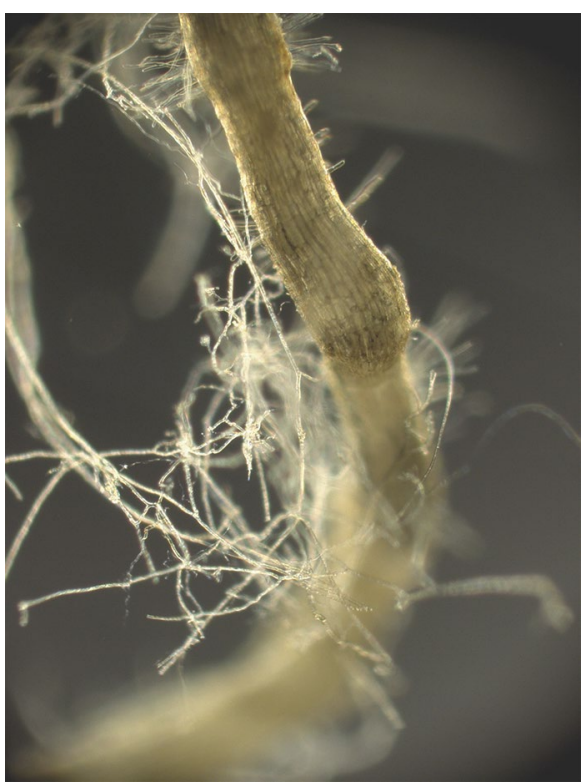

Credit: Yoshihiro Kobae

The buried is often more revealing than the displayed; this general rule may also apply to plants. A lot of the crucial nutritional action is hidden from view, far even from the roots, happening deep in the soil we walk on. Indeed, fungi form symbiosis with most plants, producing a vast underground interconnected network of filamentous hyphae that makes it possible to forage for resources in distant and richer parts of the soil. These nutrients are then exchanged for necessary plant sugars in a mutualistic interaction.

Until now, it has been extremely difficult to track this trade. In a study published last month, Toby Kiers and colleagues have developed an ingenious new method to exquisitely visualize and quantify phosphorus (P) dynamics from an exogenous source to the fungal network and finally, to the plant roots. As an experimental system, they chose a common symbiosis between an arbuscular mycorrhizal fungus and carrot roots. Labelling two sources of $\mathrm{P}$ with nanoparticles of different colours, the authors manipulated resource distribution by creating poor and rich soil patches. When the fungus is faced with resource inequality, nutrient transfer from the fungus to the roots increases and hoarding by the fungus is dramatically reduced. Furthermore, within the fungal network $\mathrm{P}$ is rebalanced by redistribution from rich to poor regions.

While the occasional use of human economics vocabulary might frighten the reader (maybe because there is no intentionality in the behaviour of a fungus), this study opens many questions that the new approach will help to answer. How can fungi coordinate nutrient transport over long distances on a decentralized hyphae network? The molecular signals and mechanisms involved are unknown at this stage. As plantfungi symbiotic interactions are essential for most plant life on Earth, from forests to crop fields, these are significant questions coming from a hidden world.

Guillaume Tena

Published online: 3 July 2019

https://doi.org/10.1038/s41477-019-0480-2 